\title{
The Multilingual Assessment Instrument for Narratives (MAIN): Adding Kam to MAIN
}

\author{
Wenchun Yang \\ The Hong Kong Polytechnic University
}

\author{
Angel Chan \\ The Hong Kong Polytechnic University
}

\section{Natalia Gagarina}

Leibniz-Zentrum Allgemeine Sprachwissenschaft (ZAS)

This paper introduces the Kam version of the Multilingual Assessment Instrument for Narratives (LITMUS-MAIN). Kam is a minority language in southern China which belongs to the Kam-Tai language family and is spoken by the Kam ethnic minority people. Adding Kam to MAIN not only enriches the typological diversity of MAIN but also allows researchers to study children's narrative development in a sociocultural context vastly distinctly different from the frequently examined WEIRD (Western, Educated, Industrialized, Rich, and Democratic) societies. Moreover, many Kamspeaking children are bilingual ethnic minority children who are "left-behind" children living in Mainland China, growing up in a unique socio-communicative environment.

\section{Introduction}

Bilingualism is extensive all over the world. It has been estimated that more than half of the world's population is bilingual (Grosjean, 2010). One long-standing challenge faced by researchers and practitioners working with bilingual children has been differentiating children with and without Developmental Language Disorder (DLD). Recently, the European Cooperation in Science and Technology COST Action IS0804 "Language Impairment in a Multilingual Society: Linguistic Patterns and the Road to Assessment" (2009-2013) was carried out to address this challenge (see Armon-Lotem, de Jong \& Meir, 2015). Its aim was to develop appropriate tools to assess the linguistic and cognitive abilities of bilingual children 
with and without language impairment speaking different pairs of languages. One of the tools developed within this action was the Language Impairment Testing in Multilingual Settings Multilingual Assessment Instrument for Narratives (LITMUS-MAIN, hereafter MAIN; Gagarina et al. 2012; 2015; 2019). MAIN was developed to further our understanding of children's development of narrative abilities. Thus far, it has been adapted or is undergoing adaption to over 70 languages to assess monolingual and bilingual children's narrative skills around the world. MAIN is not only suitable for children aged 3 to 12, but can also be used with adults (see Gagarina, Bohnacker \& Lindgren, 2019). It consists of four parallel picturebased stories and scripts: Cat, Dog, Baby Birds and Baby Goats. The stories are controlled for cognitive and linguistics complexity, allowing us to conduct dual language testing on bilinguals and draw comparisons between different bilingual children from diverse language and cultural backgrounds. MAIN tests macrostructure (following Stein \& Glenn, 1979; and Westby, 2005's story grammar model) and microstructure (the use of specific language elements). It also allows one to assess Internal State Terms (ISTs). Each story contains three episodes and each episode contains five macro-structural components: Goal (G), Attempt (A), Outcome (O), IST as an initiating event, and IST as a reaction to the outcome. Macrostructure can be assessed quantitatively by calculating the total number of components expressed in a story (i.e. story structure) and qualitatively by computing the combination of episode components (i.e. story complexity, e.g., GA, AO, GAO etc., see Gagarina et al., 2015 for a more detailed description).

Thus far, MAIN has predominantly been adapted to European languages. Adding Kam, a Kam-Tai language, which is distinctly different typologically, will widen the empirical coverage of MAIN.

\section{An overview of Kam and the reasons for adapting MAIN to Kam}

Kam is a minority language in southern China, spoken by the Kam ethnic minority people. According to the 2010 census of Peoples' Republic of China (NBS, 2010), the population of the Kam people is about 2.87 million, and they mainly live in the south and southwest of China, in the Guizhou and Hunan provinces and Guangxi Zhuang Autonomous Region. Kam belongs to the Kam-shui branch of the Kam-Tai language family, ${ }^{1}$ which is typologically different from the Indo-European languages in phonetics, phonology, morphology and syntax. For example, Kam has a complex and conservative tone system with up to 15 phonetic tones ( $\mathrm{Wu}, 2018)$. Kam permits both subjects and objects to be omitted. Referring expressions are also different in Kam compared to English and many other European languages; since Kam lacks an indefinite article, bare nominals (i.e. nominals without any determiner) are used to introduce new referents as seen in (1) (extracted from a story by a Kam speaker).

\footnotetext{
${ }^{1}$ The genetic relationship of Kam-Tai languages is still debated with some researchers arguing that they are part of Sino-Tibetan languages ( $\mathrm{Li}, 1965,1973)$ and others arguing that they belong to the Austronesian languages (Benedict, 1942, 1975).
} 


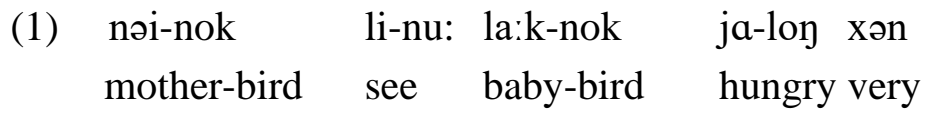

'A mother bird saw that her baby bird was very hungry.'

Thus far, a large proportion of participants assessed with MAIN come from the WEIRD (Western, Educated, Industrialized, Rich, and Democratic) societies (Henrich et al., 2010). Most minority children in Mainland China including Kam children live in relatively less developed provinces and come from low-SES families without sufficient parental care and support (Chen et al., 2018). Many of these ethnic minority children are so-called "left-behind" children because they remain in the rural areas when their parent(s) move to cities for employment. These children are therefore primarily taken care of by their grandparent(s), relatives or family friends who often have low education levels and do not interact with these children in the same way as parents would. Currently, little is known about the impact of this unique social-communicative environment on the language development of these 'left-behind' children. Raised in an environment that is distinctly different both linguistically and socioculturally from the frequently studied WEIRD populations, these ethnic minority children in Mainland China are important to investigate because such studies might shed light on the putative universals in language acquisition, which have never been examined in this language constellation and sociocultural context.

\section{Adapting MAIN to Kam}

The Kam MAIN version was adapted from the revised English MAIN (Gagarina et al., 2019) following the guidelines by Bohnacker and Gagarina (2019). The adaptation procedure was as follows. In the first phase, the first author (Yang), a near-native speaker of Kam with a $\mathrm{PhD}$ degree in linguistics and language acquisition, translated the MAIN protocol into Kam under the supervision of the second author (Chan; a developmental psycholinguist with a speech therapist qualification serving as a professorial faculty member at a university in Hong Kong), and the third author (Gagarina; the original author leading the development of MAIN and its cross-linguistic adaptations). In the second phase, the translations were carefully proofread for consistency and accuracy by three native speakers of Kam, all of whom have college-level education. The first author then incorporated the suggestions for changes and proofread the entire manuscript again. The back translations were compared and the final version was established.

The Kam MAIN has also been piloted with thirteen native adult speakers. The pilot study confirmed that the MAIN stories and pictures are culturally appropriate for minority Kam speakers: no participants reported any cultural inappropriateness in the stories or pictures. The protagonists of the four stories: a boy, a dog, a cat, goats and birds and other animals such as a mouse and a butterfly are common in Kam villages and the Kam speakers had no difficulties in recognizing these animals in the pictures. The plot and setting of the stories are also appropriate 
to the Kam culture. All Kam-speaking adult participants could readily narrate the MAIN stories without noticeable difficulties.

\section{$4 \quad$ Final remarks}

To conclude, the Kam MAIN can be used to assess narrative comprehension and production abilities in monolingual and bilingual Kam speakers. We here publish (i) the assessment protocol, which contains the four story scripts together with the scoring forms and instructions for administration; and (ii) this introductory paper that provides key information of our adaptation process, with the intention to make this new tool freely accessible to researchers and clinicians, both locally and internationally. Studies that make use of this tool should cite both the assessment protocol and this introductory article as follows:

- Gagarina, N., Klop, D., Kunnari, S., Tantele, K., Välimaa, T., Bohnacker, U. \& Walters, J. (2019). MAIN: Multilingual Assessment Instrument for Narratives - Revised. Materials for use. ZAS Papers in Linguistics, 63. Kam version. Translated and adapted by Yang, W.C., Chan, A. \& Gagarina, N.

- Yang, W.C., Chan, A. \& Gagarina, N. (2020). The Multilingual Assessment Instrument for Narratives (MAIN): Adding Kam to MAIN. ZAS Papers in Linguistics, 64, 147-151.

\section{$5 \quad$ References}

Armon-Lotem, S., de Jong, J., \& Meir, N. (Eds.). (2015). Assessing multilingual children: Disentangling bilingualism from language impairment. Bristol: Multilingual Matters.

Benedict, P. K. (1942). Thai, Kadai, and Indonesian: A new alignment in Southeastern Asia. American Anthropologist, 44(4), 576-601.

Benedict, P. K. (1975). Austro-Thai language and culture with a glossary of roots. New Haven: Human Relations Area Files Press.

Bohnacker, U., \& Gagarina, N. (2019). Background on MAIN - Revised, how to use it and adapt it to other languages. ZAS Papers in Linguistics, 63, iv-xii.

Chen, S., Lawrence, J. F., Zhou, J., Min, L., \& Snow, C. E. (2018). The efficacy of a school-based book-reading intervention on vocabulary development of young Uyghur children: A randomized controlled trial. Early Childhood Research Quarterly, 44, 206-219.

Gagarina, N., Bohnacker, U. \& Lindgren, J. (2019). Macrostructural organization of adults' oral narrative texts. ZAS Papers in Linguistics, 62, 190-208.

Gagarina N., Klop, D., Kunnari S., Tantele K., Välimaa T., Balčiūnienė I., Bohnacker, U., \& Walters, J. (2012). MAIN: Multilingual Assessment Instrument for Narratives. ZAS Papers in Linguistics, 56.

Gagarina, N., Klop, D., Kunnari, S., Tantele, K., Välimaa, T., Balčiūnienė, I., Bohnacker, U., \& Walters, J. (2015). Assessment of narrative abilities in bilingual children. In Armon-Lotem, S., Jong, J. d. \& Meir, N. (Eds.), 
Assessing multilingual children: Disentangling bilingualism from language impairment (pp. 243-276). Bristol: Multilingual Matters.

Gagarina, N., Klop, D., Kunnari, S., Tantele, K., Välimaa, T., Bohnacker, U. \& Walters, J. (2019). MAIN: Multilingual Assessment Instrument for Narratives. Revised version. ZAS Papers in Linguistics, 63.

Grosjean, F. (2010), Bilingual: Life and reality. Cambridge, MA: Harvard University Press.

Henrich, J., Heine, S., \& Norenzayan, A. (2010). The weirdest people in the world? Behavioral and Brain Sciences, $33(2-3), 61-83$.

Li, F. K. (1965). The Tai and the Kam-Sui languages. Lingua, 14, 148-179.

Li, F. K. (1973). Languages and dialects of China. Journal of Chinese Linguistics, 1(1), 1-13.

NBS (National Bureau of Statistics). (2010). Sixth national population census of the People's Republic of China. China Statistical Press: Beijing.

Stein, N. L., \& Glenn, C. G. (1979). An analysis of story comprehension in elementary school children. In R. O. Freedle (Ed.), Discourse processing: Multidisciplinary perspectives (pp. 53-120). Norwood, NJ: Ablex.

Westby, C. E. (2005). Assessing and facilitating text comprehension problems. In H. Catts \& A. Kamhi (Eds.), Language and reading disabilities (pp. 157-232). Boston: Allyn \& Bacon.

Wu, M. X. (2018). A grammar of Sanjiang Kam. Muenchen: LINCOM Europa. 